\title{
On the Formation and Development of Tropical Cyclones ${ }^{1}$
}

\author{
H. LANDERS \\ Florida State University \\ (Manuscript received 23 July 1962, in revised form 27 November 1962)
}

\begin{abstract}
Research in tropical meteorology has revealed that the average easterly winds found in tropical regions are perturbed by large numbers of synoptic-scale disturbances. The mechanisms responsible for the initiation of such disturbances and the intensification of some of them into hurricanes may be considered as a problem of the instability of the basic easterly current and its perturbations. This study presents results of investigations into this problem for the region of the tropical North Atlantic Ocean, Caribbean Sea and Gulf of Mexico. Initial wave formation and subsequent intensification to the vortex stage are emphasized. Empirical relationships between the vertical (geostrophic) wind shear and wave or vortex intensification are consistent with the predictions of the Bjerknes-Homboe theory modified for easterly flow. The 1000-500mb shear (thickness) patterns were used as a measure of the depth of the easterlies. A net easterly shear in this layer was found to be favorable for intensification and a net westerly shear unfavorable. Empirical relationships between sequences of events in the westerlies and associated variations in the structure and position of sub-tropical anticyclones are found to predict the time and place of formation of one class of tropical disturbances. Methods of forecasting initial development and changes in intensification of tropical disturbances based on the combined empirical and theoretical work were developed and tested on independent data. The results were quite favorable, on the whole, although the verification system is not completely free of subjectivity.
\end{abstract}

\section{Initiation of tropical cyclones}

A survey of the historical series of $500-\mathrm{mb}$ charts (U. S. Weather Bureau, Synoptic Weather Maps) during several tropical storm seasons suggested that a few days prior to each storm a rather vigorous trough in the westerlies moved eastward from the North American continent to the Atlantic Ocean. Evaluation of the zonal index data for the years 1952-55 in the section $20 \mathrm{~W}$ to $140 \mathrm{~W}$ during the tropical storm seasons, indicated that a marked increase in westerlies $(30-70 \mathrm{~N})$ frequently was followed by a hurricane two or three days later. Curves of the ratio: $r=I_{(30-45 N)} / I_{(30-70 N)}$ (where the I's are zonal index measures) were plotted for the four years. The time of the first Weather Bureau advisory was used as a reference time $=0$ for Fig. 1 . Segments of $r$ curves from 6 days prior to and 5 days following the first advisory were added for 33 storms and the average $\tilde{r}$ obtained. Fig. 1 shows that $r$ increased until about $2 \frac{3}{4}$ days before storm occurrence, then decreased rapidly until $\frac{1}{4}$ day after occurrence, and then increased again to normal values. Individual cases showed $r$ varying from 20 to 40 per cent and averaging 30 per cent. Average value for $\vec{r}$ in Fig. 1 has been arbitrarily set at zero. The total variation in $r$ is 12 per cent

1 This research was sponsored partly by the Geophysics Research Directorate, Air Force Cambridge Research Laboratories, and by the Southern Fellowships Fund. The principal sponsor was the
U. S. Weather Bureau.

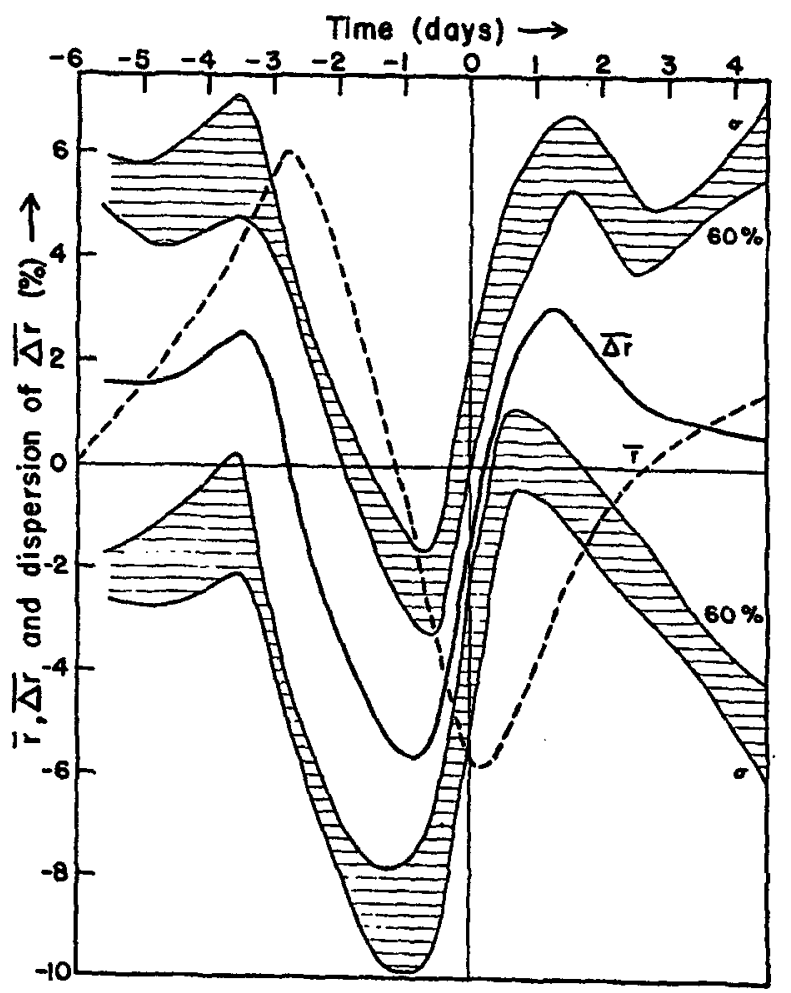

Fig. 1. $\vec{r}, \overline{\Delta r}$ and dispersion of $\overline{\Delta r}$ vs. time for a sample of 33 tropical cyclones. 
as compared with 20 per cent in individual cases. $\overline{\Delta r}$ is simply the change in $\bar{r}$ with time, 24 hours being the interval selected. $\overline{\Delta r}$ is positive where $\bar{r}$ is increasing and negative where $\bar{r}$ is decreasing and shows that the greatest change in $\bar{r}$ is the rapid decrease occurring at one day prior to storm occurrence. A measure of the dispersion of $\overline{\Delta r}$ is provided by the hatched regions. Sixty per cent of the cases fell within the inner limits of these regions. The outer limits of the hatched region represent the standard deviation $(\sigma)$.

Since the correlation between westerly zonal-index changes and time of storm formation appeared to be related directly to the eastward passage across the Atlantic of a strong $500-\mathrm{mb}$ trough, it was felt that an investigation of the behavior of the subtropical anticyclone in the North Atlantic might shed some light on the relation between easterlies and westerlies.

In 1955, several tropical storms formed east of the Lesser Antilles, and there were several major northsouth oscillations in the position of the subtropical high. After considerable experimentation, a parameter was adopted which included the 500 -mb latitude of the high, the maximum height in the high and the height gradient south of it. This parameter indicates whether the wind speeds increase south of the center of the high, provided there is little or no southward motion of the intertropical trough. It would be very desirable to use an easterly zonal index alone rather than combining it with two other factors. However, the latitude of the anticyclone and its central height both can be determined with considerably more accuracy than the height gradient (zonal index) south of the anticyclone. It follows that if the anticyclone moves southward without decreasing (or perhaps even increasing) its central height, that the height gradient in the easterlies must increase provided the trough to the south changes little in position and intensity. A detailed explanation of the computation of this parameter accompanies Fig. 17. Suffice it to say here only that small values describe optimum conditions for wave formation in the easterlies.

All of the values included in the parameter were averaged over the $25-45 \mathrm{~W}$ section because the subtropical high during the summer of 1955 was centered near $35 \mathrm{~W}$. The plot of this parameter against time is shown in Fig. 2. The minor oscillations of ordinate values of five or less are due to the inevitable uncertainties in the analyses. The large oscillations (the dips in the curve) can be related to time of wave formation resulting in tropical storms. The time of storm formation is represented by the hurricane symbol ( $\boldsymbol{5}$ ) and the longitudes of the places of the first report are given. Arrows extend backward in time to the major dips in the curve, and the longitude values near the arrowheads represent the initial positions of waves, assuming each wave and storm moved at a 10-knot speed until detected. Six major storms are related to six major dips, the storms being noted first between $42 \mathrm{~W}$ and $60 \mathrm{~W}$. The waves on which they developed are assumed to have originated between $30 \mathrm{~W}$ and $41 \mathrm{~W}$ and this coincides very well with the mean longitude of the high, $35 \mathrm{~W}$. A wave was carried for two days in July, which could have been related to the minor dip on 27 July. The Gulf-of-Mexico storms ( $87 \mathrm{~W}$ and $97 \mathrm{~W}$ ) have no related dips in the curve. The only apparent exception is Hilda which was first established as a tropical storm on 9 September at $65 \mathrm{~W}$. Careful examination of daily surface maps indicates that a wave of very large amplitude may have produced both Hilda and Flora. Flora occurred early near the upper part (northern extension) of a long trough and the upper and lower parts separated soon thereafter. The southern part could be traced westward to the position where Hilda was first reported several days later.

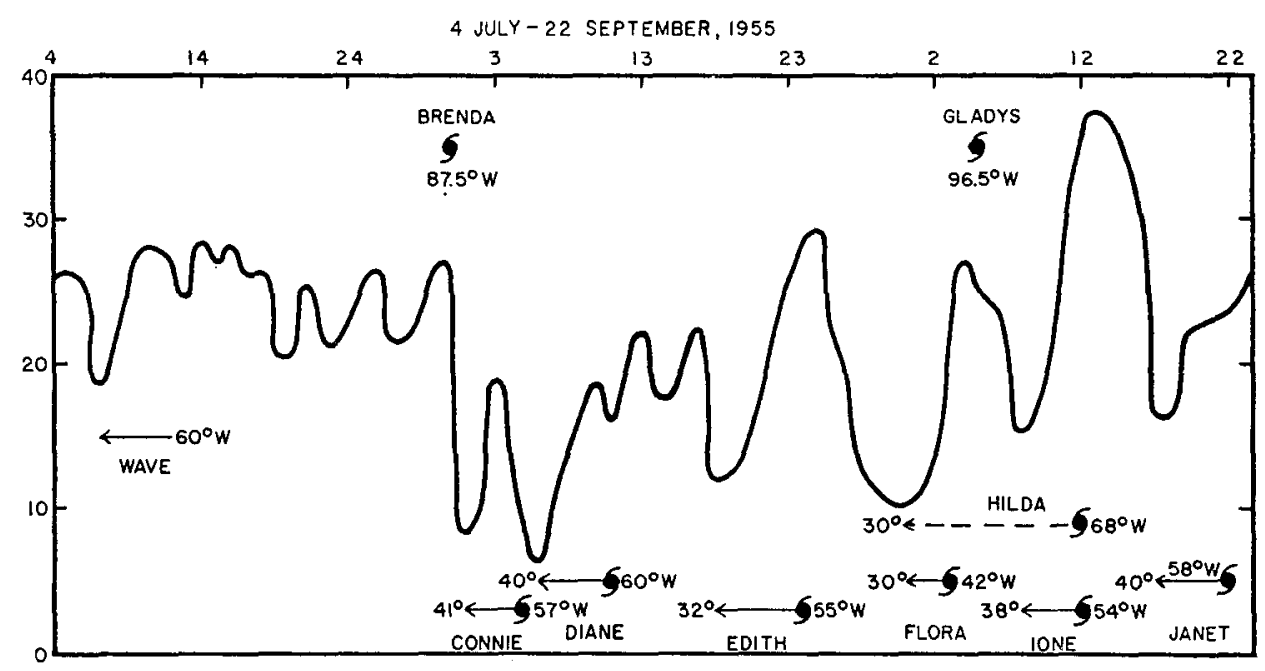

FIG._2. Parameter related to time of tropical cyclone formation vs. time (1955). 
Willett's result ${ }^{2}$ that the easterlies increased in strength in winter and shifted farther south as the westerlies strengthened and shifted southward agrees with our findings. Willett used five-day mean maps, had easterly and westerly wind belts which encircled the earth and noted the above-mentioned trend on a long term basis. Riehl, Yeh and LaSeur (1950) found that tropical storm activity was absent when the westerly momentum anomaly was far to the south but appeared to begin at about the time the momentum maximum had traveled north to the latitude of maximum westerlies. One could easily interpret their results as follows: the westerlies are far south early in the period and this is actually the time when large amplitude waves can form in the easterlies. A wave forms then and later becomes a vortex which gradually increases to typhoon intensity. The time lag was about 9 days, a reasonable period to occur between wave formation and typhoon detection in the Pacific in 1945. Namias (1950), considering half of the Northern Hemisphere, showed that curves of five-day averages of westerly wind with latitude exhibited little change in total momentum. Increased westerlies in mid-latitudes were accompanied by decreased westerlies at high latitudes and/or increased easterlies in low latitudes. LaSeur and Jordan ${ }^{3}$ found the westerlies dipped far to the south prior to an active typhoon period. An inspection of the 500-mb maps shows the large subtropical high cell in their region of computation was displaced to the south at the same time. Here the time lag was five days before the first cyclonic closed isobar appeared on the surface map, and on the sixth day the circulation was definitely present at $500 \mathrm{mb}$. In summary it can be stated that earlier results can be rendered compatible with those of the writer.

The period of 19-30 August 1955 coincides with a major cycle on the curve in Fig. 2. The three-day height changes presented in Figs. 3 and 4 were obtained from the analyses in the historical series and coincide with the greatest changes in the parameter in Fig. 2; Fig. 3 representing increasing values (20-23 August) and Fig. 4 decreasing values (25-28 August). $A_{20}$ and $\mathrm{A}_{23}$ in Fig. 3 represent the positions of the main anticyclone on 20 and 23 August. The central height value in the anticyclone decreased $80 \mathrm{ft}$ during the period but even so there is a three-day height rise of $160 \mathrm{ft}$ at $\mathrm{A}_{23}$. There is a 250 -ft height fall at $\mathrm{A}_{20}$. The large height rises at $50 \mathrm{~N}$ are a result of motion and changes in intensity of migratory features in the westerlies. In Fig. 4, a three-day height fall of $110 \mathrm{ft}$ occurred at $\mathrm{A}_{25}$ and a rise of $150 \mathrm{ft}$ at $A_{28}$. The height value at the center of the anticyclone decreased $50 \mathrm{ft}$ during the period.

\footnotetext{
${ }^{2}$ Willett, H. C., and collaborators, 1947: Final Report of the Weather Bureau-Massachusetts Institute of Technology extended forecasting project for the fiscal year July 1, 1946-June 30, 1947. Cambridge, (mimeographed) $110 \mathrm{pp}$.

${ }^{3}$ LaSeur, N. E., and C. L. Jordan, 1952: A typical weather situation of the typhoon season. University of Chicago, ONR Contract N60ri-20, 24 pp.
}

The large fall centers southwest of Spain and over Nova Scotia are caused by troughs in the westerlies which slowed and deepened. The rise center north of the Azores is due to the cut-off part of the subtropical high which lies between the two strong troughs. The rise and fall areas in the southwe:t corner of the map reflect the motion and deepening of hurricane Edith.

The 500-mb contour analyses for 19 and 28 August, Figs. 5 and 6 , coincide with times when the parameter in Fig. 2 was at a minimum. Tłe values in Table 1

TABLE 1. Comparison of instantaneous $500-\mathrm{mb}$ heights with monthly normal values.

\begin{tabular}{lccc}
\hline & \multicolumn{3}{c}{$500-\mathrm{mb}$ heights in gpf } \\
Coordinates & $\begin{array}{c}19 \text { August } \\
\text { 28 fugust } \\
\text { j955 }\end{array}$ & $\begin{array}{c}\text { August } \\
\text { normal }\end{array}$ \\
\hline $52 \mathrm{~N}, 33 \mathrm{~W}$ & 18,000 & 19,000 & 18,600 \\
$45 \mathrm{~N}, 65 \mathrm{~W}$ & 18,980 & 17,980 & 18,920 \\
$34 \mathrm{~N}, 14 \mathrm{~W}$ & 19,570 & 13,690 & 19,280 \\
$15 \mathrm{~N}, 30 \mathrm{~W}$ & 19,420 & 19,490 & 19,270 \\
\hline
\end{tabular}

illustrate the main similarities and differences in the two cases. On the 19th a strong cyclone lies southwest of Iceland and a trough extends southward along $35 \mathrm{~W}$ causing heights in the north-central Atlantic to be some $600 \mathrm{ft}$ below normal. The subtropical ridge is south of its normal position in the central Atlantic causing above normal heights in the lower latitudes. The trough on the northeastern coast of the United States is very weak and does not intensify but moves rapidly to the east-northeast. On the 28th the main Atlantic anticyclone is again south of normal jut its smaller extension northward causes the height at $52 \mathrm{~N}, 33 \mathrm{~W}$ to be $1000 \mathrm{ft}$ higher than on the 19 th. The two strong troughs on either side of the northward extension of the high show heights that are 600 to $900 \mathrm{ft}$ below normal. The cyclone south of Bermuda is hurr.cane Edith.

Here are two cases where the main cell of the subtropical anticyclonic system was displaced far to the south by vigorous cyclonic activity in the westerlies. On the 19th a single vortex-trough system was present in the mid-Atlantic while on the 28th there were two moving troughs which slowed and deepened as they moved into the normal long-wave positions.

\section{Dynamics and kinematics of the storm-breeding region}

The normal sea-level wind analysis for August is shown in Fig. 7. Original data from the Atlas of climatic charts of the oceans were used ind considered to be accurate to 5 deg and 1 knot. Actually a $1 \frac{1}{2}$ or 2 knot accuracy would produce essentially the same speed analysis as shown in Fig. 7. Careful analysis of several months of normal data indicate separate wind-speed maxima tend to occur at about $20,32,48$ and $72 \mathrm{~W}$. The ones at $20 \mathrm{~W}$ and $72 \mathrm{~W}$ car be related to land 

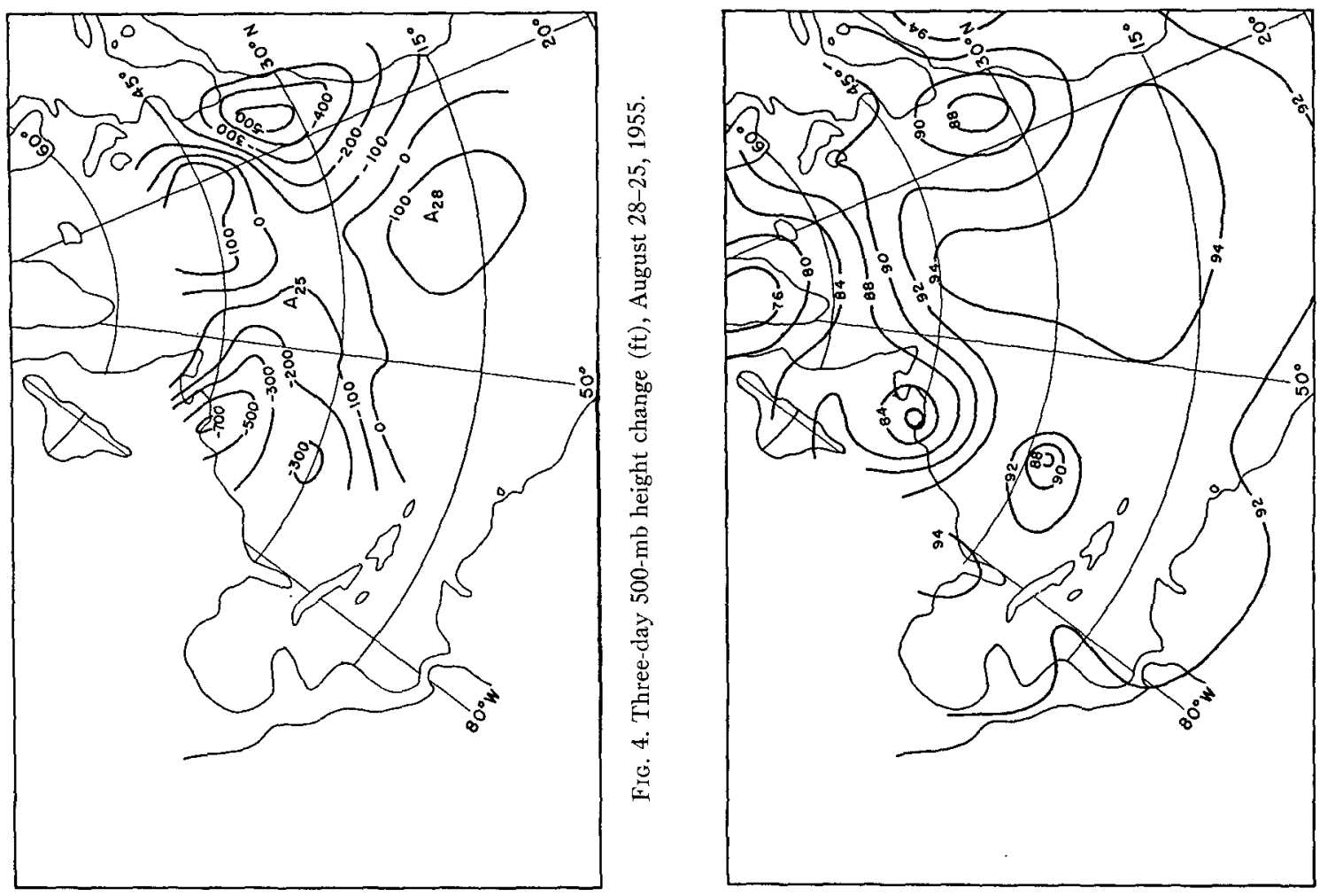

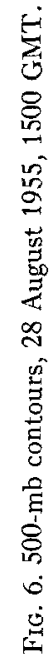
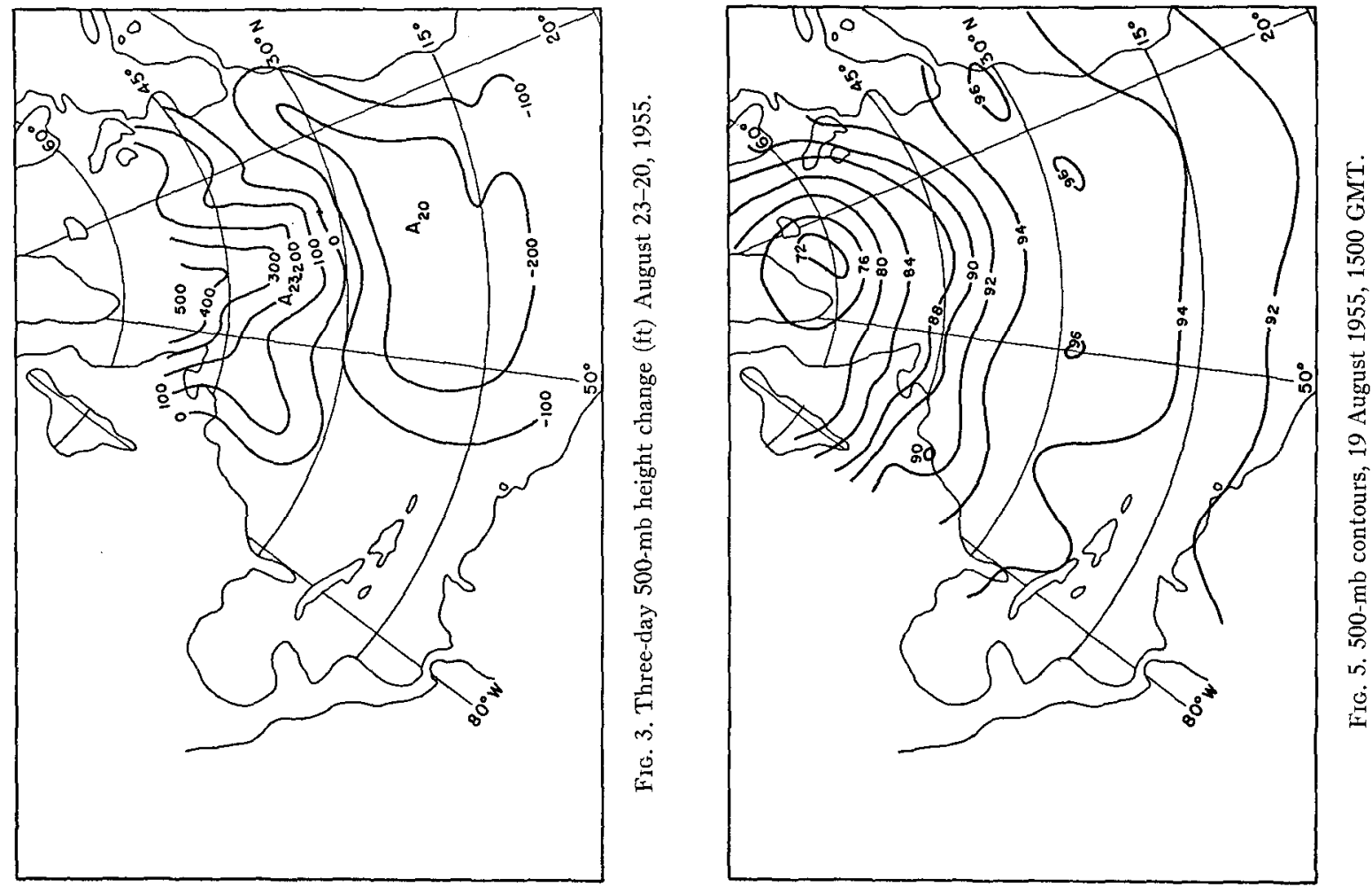


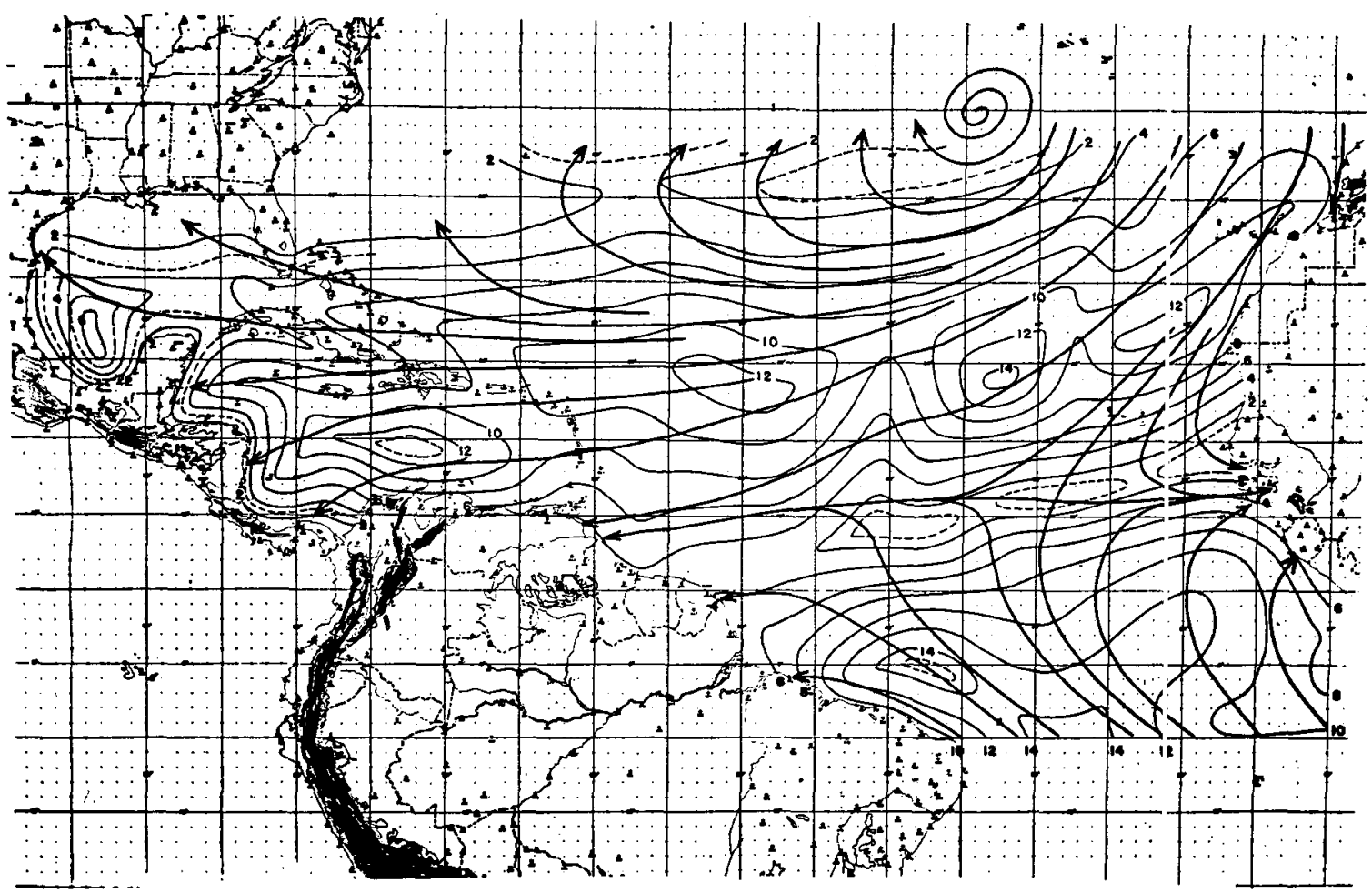

FIG. 7. August normal surface wind, streamlines and isovels (knots).

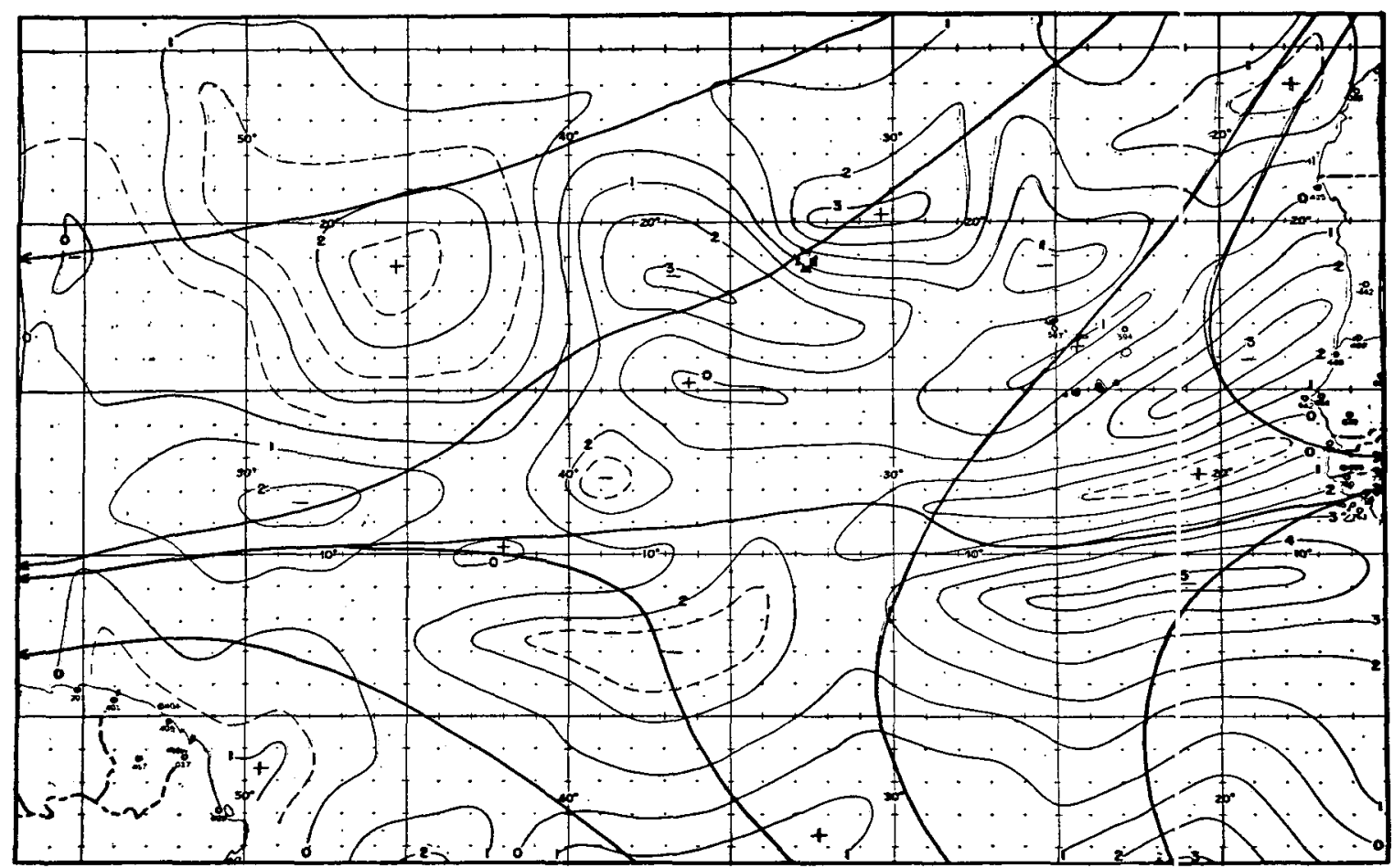

FIG. 8. $\vec{D}=\nabla_{H} \cdot \bar{V}\left(\times 2.5 \times 10^{-6} \mathrm{sec}^{-1}\right)$. 


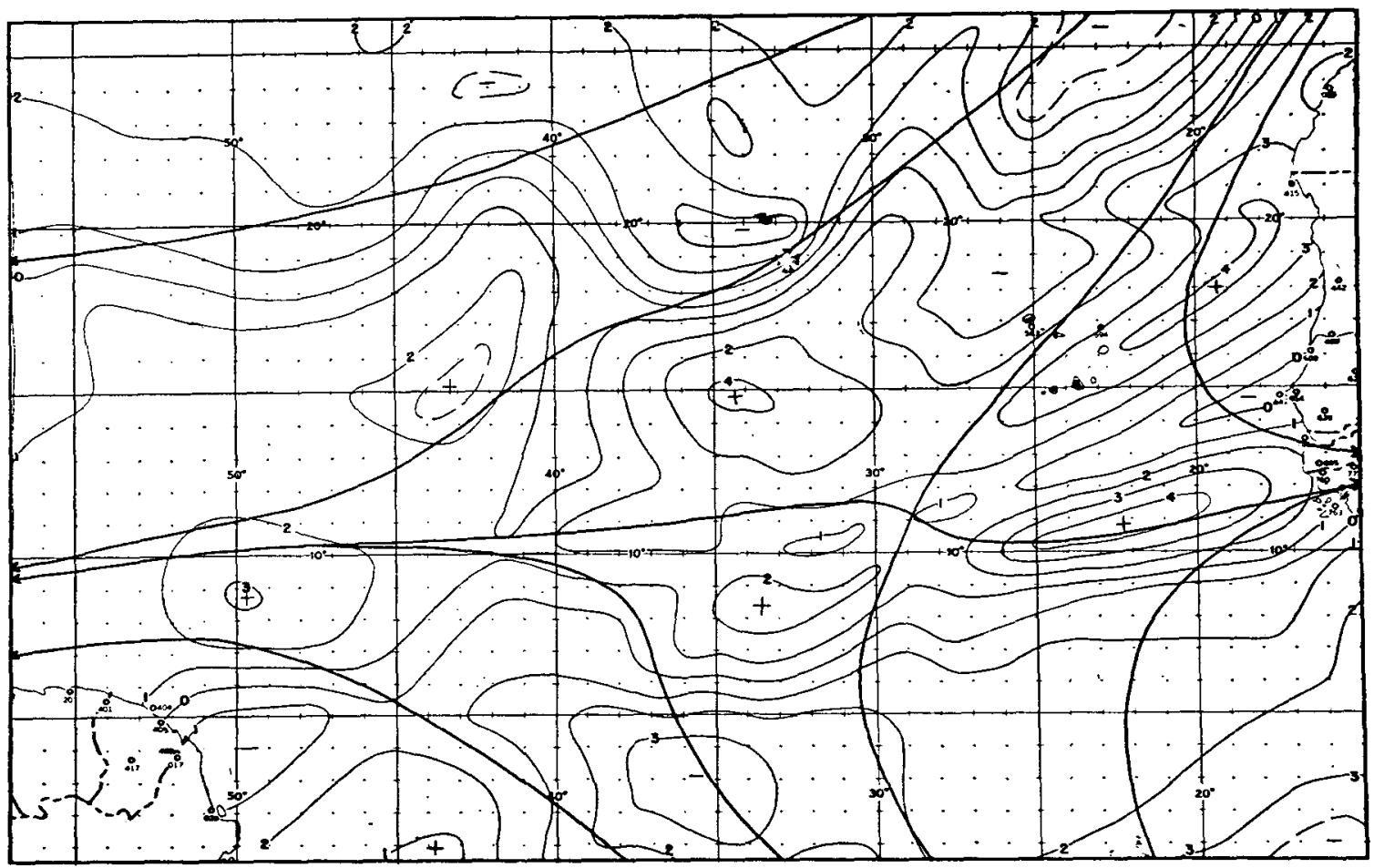

FIG. 9. $s^{\circ}\left(\times 2.5 \times 10^{-6} \sec ^{-1}\right)$.

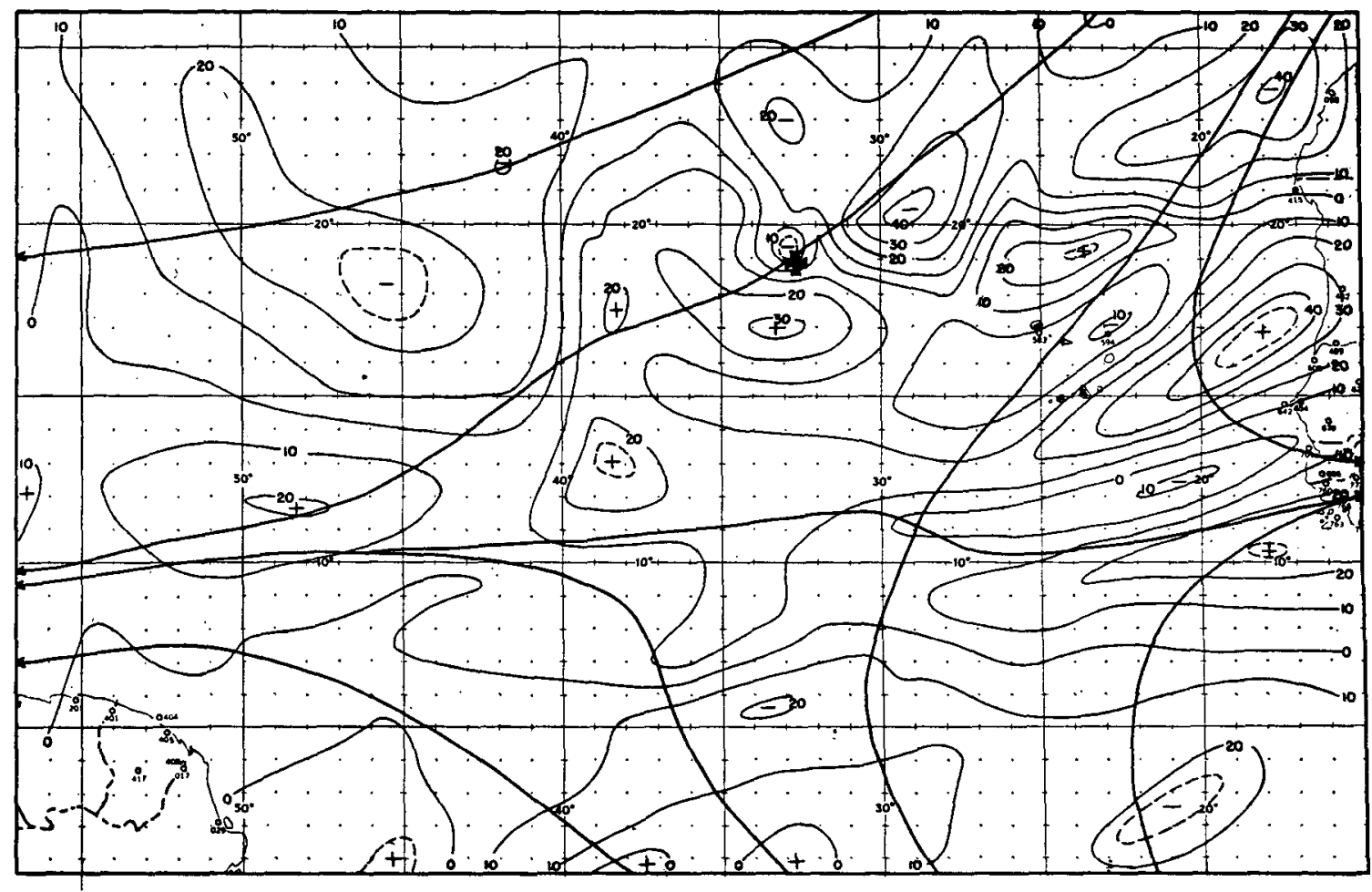

Fig. 10. $-\left(\overline{\zeta D}+\beta \bar{v}+\varepsilon \frac{\partial \bar{\zeta}}{\partial s}\right)\left(\times 10^{-11} \sec ^{-2}\right)$. 
features but not those at $32 \mathrm{~W}$ and $48 \mathrm{~W}$. The one at $32 \mathrm{~W}$ is located directly between the center of the Azores high and the col in the equatorial trough and it is postulated that it exists because of aperiodic excursions of the high southward. The speed maximum at $48 \mathrm{~W}$ could be an indirect result of the one at $32 \mathrm{~W}$.

It is impossible to obtain a detailed instantaneous map so the normal map was used in an attempt to gain some information concerning the make-up and stability of this pattern through an application of the vorticity equation.

It was shown by Landers ${ }^{4}$ that in similar tropical latitudes in the Pacific in summer, several terms in the vorticity equation are small enough to be excluded. A simplified but meaningful form of the equation can be written.

$$
\frac{\delta \zeta}{\delta t}=-\left(\zeta_{a} D+\beta v+c \frac{\partial \zeta}{\partial s}\right)
$$

Here, $\zeta$ and $\zeta_{a}$ are relative and absolute vorticity, respectively, $D=\nabla_{H} \cdot \mathrm{V}, \beta=\frac{\partial f}{\partial y}, c$ is horizontal wind speed, $v$ is the $y$ component of $c$ and $s$ is the direction of horizontal streamlines.

Since we are dealing with a normal map we can write (1) as

$$
\frac{\overline{\delta \zeta}}{\delta t}=-\bar{\zeta}_{a} \bar{D}-\overline{\beta v}-\bar{c} \frac{\overline{\partial \zeta}}{\partial s} .
$$

The basic components of the terms in (1) can be considered to be composed of time-averaged values plus deviations and substituted in (2). When (2) is expanded and terms rearranged, we have,

$$
\overline{\frac{\delta \zeta^{\prime}}{\delta l}}+\overline{\mathbf{V} \cdot \nabla \zeta^{\prime}}+\overline{\mathbf{V}^{\prime} \cdot \nabla \zeta^{\prime}}=-\overline{\mathbf{V} \cdot \nabla \bar{\zeta}}-\overline{\bar{\zeta}} \overline{\bar{D}}-\overline{\beta \bar{v}}-\overline{\zeta_{a}^{\prime} D^{\prime}}
$$

Now since $\frac{d \zeta^{\prime}}{d t}=\frac{\delta \zeta^{\prime}}{\delta t}+\mathrm{V} \cdot \nabla \zeta^{\prime}=\frac{\delta \zeta^{\prime}}{\delta t}+\overrightarrow{\mathbf{V}} \cdot \nabla \zeta^{\prime}+\mathbf{V}^{\prime} \cdot \nabla \zeta^{\prime}$ we can replace the left side of (3) with $\frac{\overline{d \zeta^{\prime}}}{d t}$ and change $\overline{\nabla \cdot \nabla \bar{\zeta}}$ to scalar form. This yields

$$
\overline{\frac{d \zeta^{\prime}}{d t}}=-\left[\overline{\bar{\zeta}} \overline{\bar{D}}+\overline{\beta \bar{v}}+\overline{\bar{\partial}} \frac{\bar{\partial} \bar{\zeta}}{\partial s}\right]-\overline{\zeta_{a}^{\prime} D^{\prime}}
$$

It is possible to compute the three terms in the bracket in (4) from the normal map. Isogons were constructed to supplement the speed field shown in Fig. 7, and divergence and vorticity were computed. The windspeed maximum of principal interest (Maltese Cross) and a few key streamlines have been transferred from

\footnotetext{
${ }^{4}$ Landers, H., 1950: Unpublished Master's Thesis. Dept. of Meteor., U.C.L.A., 21 pp.
}

Fig. 7 to these and later charts so that continuous crossreference will not be necessary.

The pattern of divergence, Fig. 8 , around the windspeed maximum $\left(32 \frac{1}{2} \mathrm{~W}, 19 \mathrm{~N}\right)$ is mainly speed divergence, positive upstream and neçative downstream with maximum values of $8 \times 10^{-6} \mathrm{sec}^{-1}$. This is about half the average value found in an instantaneous Pacific case (See footnote 3), however, it is quite significant for a 50-yr normal value.

A zero line of relative vorticity, Jig. 9, lies close to the axis of maximum wind-speed, brcause the streamlines lie along the maximum and almost all of the vorticity is in the wind shear. A small amount of vorticity is due to curvature, however, and a slight wave appears in the streamlines (and isogens), with the ridge and trough found in regions of anticyclonic and cyclonic vorticity, respectively. This wave afpeared in the freehand streamlines as well as the more detailed isogon patterns in August, September, and, to a lesser extent, in October. The streamline which passes through the Maltese Cross is the one of maximum wave amplitude.

It should be stressed that the vorticity and divergence of the normal wind field have some significance synoptically because they represent averages of the vorticity and divergence patterns which are found in significant and repetitive synoptic patterns.

The $\bar{\zeta}_{a} \bar{D}$ term has the sign of the divergence and values of $+50 \times 10^{-11} \mathrm{sec}^{-2}$ and $-35 \times 10^{-11} \mathrm{sec}^{-2}$ occur up- and downstream, respectively, of the wind-speed maximum. The $\frac{\bar{c} \partial \bar{\zeta}}{\partial s}$ term has values of $-30 \times 10^{-11} \mathrm{sec}^{-2}$ and $+20 \times 10^{-11} \mathrm{sec}^{-2}$ which coincide roughly with the $\bar{\zeta}_{a} \bar{D}$ centers of opposite sign so that :ome compensation between the two terms is noted. The $\beta \bar{v}$ term contributed very little west of $35 \mathrm{~W}$, but added as much as $-5 \times 10^{-11}$ $\mathrm{sec}^{-2}$ to the vorticity advection t3rm in the region upstream from the wind maximum.

Fig. 10 shows $-\left[\bar{\zeta}_{a} \bar{D}+\beta \bar{v}+\bar{c} \frac{\delta \bar{\zeta}}{\delta s}\right]$. The signs have been reversed so that positive values mear positive $\frac{d \zeta^{\prime}}{d t}$ contributions and vice versa. The eddy vorticity-divergence term, $\zeta_{a}^{\prime} D^{\prime}$, cannot be evaluated directly but can be treated qualitatively. Synoptic evidence is at hand (Landers, 1950) to suggest that $\zeta^{\prime}$ and $D^{\prime}$ are of the same order of magnitude as $\bar{\zeta}$ anc. $\bar{D}$. In the initial stages of development of systems $\zeta_{1}^{\prime} D^{\prime}$ will be smaller than $\bar{\zeta}_{a} \bar{D}$ and in later stages it will te larger. The model "easterly wave" and to some extent the "equatorial wave" tend to show wind speed ma:xima near the ridge lines and minima near the trough lines. A similar arrangement appears in the normal ; treamlines and the speed distribution in the northeast trades in August, Fig. 7. 


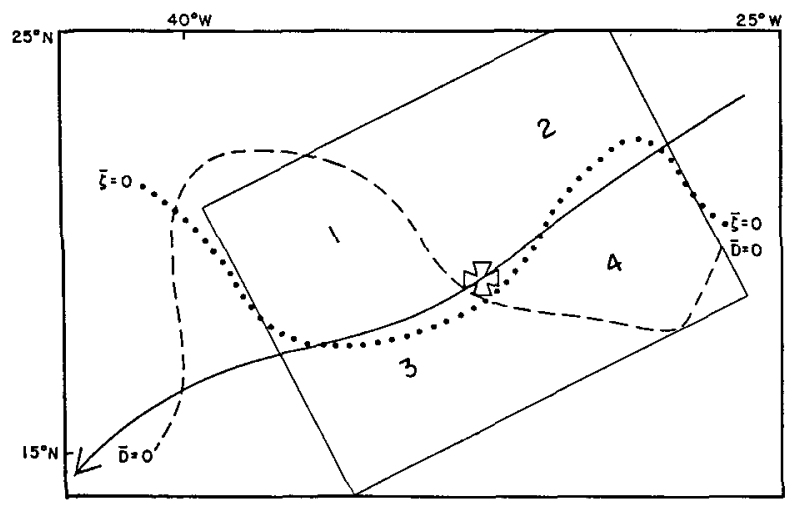

Fig. 11. Zero lines of $\bar{D}$ and $\bar{\zeta}$ and streamline in region of wind-speed maximum.

It has been postulated from empirical evidence that the existence of the wind-speed maximum at $32 \frac{1}{2} \mathrm{~W}, 19 \mathrm{~N}$ is due to aperiodic increase in pressure gradient in that locality. If this is true, in a given case of development the wind speeds increase "in situ" at the maximum and increase less or perhaps remain unchanged at the downstream speed minimum. Fig. 11 shows the part of the northeast trades which embodies the principal windspeed maximum, the downstream minimum and the wave in the normal streamlines. Zero lines of $\bar{D}$ (dashed) and $\bar{\zeta}$ (dotted) are shown and the area of the windspeed maximum has been enclosed by a rectangle which is divided into four parts. The divergence $(\bar{D})$ is nearly all in the speed term so its zero lines lies nearly normal to the streamlines at the place of maximum wind speed. The vorticity $(\bar{\zeta})$ is nearly all in the shear term so its zero line coincides very closely with the streamline passing through the speed maximum.

The $\bar{\zeta}_{a} \bar{D}$ term has the same zero line as $\bar{D}$ and has the sign of $\bar{D}$ because $f$ is considerably larger than $\bar{\zeta}$ at these latitudes. Therefore $\bar{\zeta}_{a} \bar{D}$ is positive in regions 2 and 4 and negative in 1 and 3 . If we assume that a wind-field pattern like the normal one exists on a given day as a "temporary" steady state and then increase the speed at the Maltese Cross to, say, double the original values, we can reason as follows about $\zeta^{\prime}$ and $D^{\prime}$. The divergence and relative vorticity will increase so that in the early part of development $\zeta^{\prime}$ and $D^{\prime}$ will have the same signs as $\bar{\zeta}$ and $\bar{D}$, respectively. Since $\zeta_{a}^{\prime}$ at any point is simply $\zeta^{\prime}$, the subscript $(a)$ can be dropped. In region $1, \zeta^{\prime}$ and $D^{\prime}$ will both be negative so $\zeta^{\prime} D^{\prime}$ is positive and therefore opposite to $\bar{\zeta}_{a} \bar{D}$ which is negative. In region $2, \xi^{\prime}$ will be minus and $D^{\prime}$ plus so $\zeta^{\prime} D^{\prime}$ is negative and opposite $\bar{\zeta}_{a} \bar{D}$. In region $3, \zeta^{\prime}$ will be plus and $D^{\prime}$ minus so that $\zeta^{\prime} D^{\prime}$ will be minus as is $\bar{\zeta}_{a} \bar{D}$. In region $4, \zeta^{\prime}$ and $D^{\prime}$ will both be plus so $\zeta^{\prime} D^{\prime}$ is plus as is $\bar{\zeta}_{a} \bar{D}$

We can consider how $\zeta^{\prime} D^{\prime}$ can change the field of $-\left[\bar{\zeta}_{a} \bar{D}+\beta \bar{v}+\bar{c} \frac{\partial \bar{\xi}}{\partial s}\right]$ near the wind maximum. South of the streamline through the wind maximum the negative area in Fig. 11 corresponding to region 4 and the positive area corresponding to 3 will both increase. The values in regions 1 and 2 will decrease. Particles moving into region 4 from the northeast will gain anticyclonic vorticity rapidly until they reach the zero line which lies between 18 and $19 \mathrm{~N}$, and 29 and 32W (Fig. 11). Here the sign of $\frac{\overline{d \xi^{\prime}}}{d t}$ changes to cyclonic and remains so until the next zero line is reached along the $40 \mathrm{~W}$ meridian. Presumably $\zeta^{\prime}$ will reach a minimum (anticyclonic maximum) at the first zero line and a maximum (cyclonic) at the second. It is quite significant that ridge and trough lines in the normal streamlines coincide closely with these zero lines.

It can be concluded that such a pattern (Fig. 7) leads to wave formation and that the streamline amplitude will be some function of the speed differences that exist between the speed maximum and its environs. It can also be concluded that the divergence is the big factor in creating or dissipating vorticity. Initially the disturbance is in the speed field only but increasing the maximum speed soon causes a perturbation to appear in the streamlines, the cyclonic part of which would appear near $40 \mathrm{~W}, 15 \mathrm{~N}$ if the speed maximum was in the August normal position. Whether or not the disturbance develops further will depend on the general synoptic pattern and the thermal stability. The result that early development should take place south of the wind-speed maximum in a region of cyclonic vorticity may be related to the observed fact that easterly waves generally appear more pulse-like than wave-like with only the cyclonic part being well developed.

There are several ways of showing that a similar wind field in an instantaneous case will tend to produce a wave in the flow pattern. For example, Newton (1959) showed that speed maxima in the westerlies tended to create perturbations and that the ridge coincided with the speed maximum and the troughs with the speed minima which lay up- and downstream of the maximum. However, one cannot produce a synoptic map of the region of interest because scarcity of reports makes a wind speed analysis impossible. The only direct evidence that a definite speed maximum exists is the normal map which has been used here. 


\section{Development of tropical waves and vortices}

Researchers and practitioners are aware of the fact that deep low-level easterlies are favorable for tropical cyclonic activity while the intrusion of westerlies to low latitudes and to fairly low levels is unfavorable. J. Bjerknes (1957) recently has given some theoretical strength to the observed facts by applying to the easterlies a theory of waves and cyclones which he and J. Holmboe developed originally for the mid-latitude westerlies in 1944.

The convergence-divergence arrangement is the same for a wave in the easterlies as for the westerly wave but since the wave is now moving toward the west, the lowlevel (rather than the upper level) divergence pattern must control the sea-level pressure changes if the wave is to develop. A relatively high level of non-divergence is needed for a developing wave and this will occur when the easterly flow extends to fairly high levels. The trough line must tilt eastward with height to insure a pressure fall at the trough line itself and this tilt is normally observed. However, a pronounced easterly tilt of the trough with height is not a necessary condition for development. Strong pressure falls and low-level divergence ahead (west) of the trough and strong pressure rises and low-level convergence behind (east of) it will set up the vertical motion pattern which results in intensification and ultimately in sea-level pressure falls at the trough line or vortex center.

Twenty-five hurricanes were officially named in the Atlantic-Caribbean region in 1952, 1953 and 1955. Two (Jan. 1955, May 1953) were eliminated because they were not true tropical storms and this left a sample of 23. The charts needed for the application (the historical map series) were not available for the 1954 storm season so that year was not included. Thickness analyses for the 1000-500 mb layer were performed for a period of three or four days at, and just prior to, the time of appearance of each of the 23 cyclonic disturbances. Such analyses are based on the geostrophic wind approximation and this approximation has been used to within $10 \mathrm{deg}$ lat. of the equator. A comparison of the geostrophic and gradient thermal winds (Forsythe, 1945 ) in the area showed the difference between the two to be extremely small. The surface and $500-\mathrm{mb}$ maps have been treated as synchronous although the $500-\mathrm{mb}$ chart is $2 \frac{1}{2}$ hours later than the surface map. Any error due to time difference is overshadowed greatly by uncertainties in the analyses due to scarcity of data.

Fig. 12 illustrates the procedure that was followed after the thickness analysis was completed. The regions of easterly shear (1000-500 mb) which occurred over water and between 10 and $35 \mathrm{~N}$ were outlined with closed curves (dashed lines). The time is the day before a cyclonic disturbance appeared. The position of the disturbance at sea-level on the next day is shown by the number 1 , the next three $24-\mathrm{hr}$ positions being marked
2,3 and 4 . The path from 1 to 4 was $c$ rawn (dotted line) and extrapolated backwards to the time of the analysis, marked $(0)$. The $(0)$ position lies in the large region of easterly shear. The three other easierly shear regions shown are too small to be of consequence in this case. Isopleths are labeled in hundreds o: geopotential feet of thickness, and warm (w) and colc (c) regions in the mean virtual temperature field have been indicated.

The most striking result obtained from the thickness analyses and superposition of in tial cyclonic disturbance locations was that all 23 ropical storms examined originated in a region of light to strong easterly shear. With no exceptions in a 3-yr sample, one can state rather positively that it is a necessary condition for storm development from a wave tc vortex stage that there be easterly geostrophic wind-shear between 1000 and $500 \mathrm{mb}$. It is, of course, not a sufficient condition since regions of easterly shear exist on days which are not followed by the occurrence of a cyclonic vortex. Eleven other cases were similar to the one shown in Fig. 12, having only one important region of easterly shear. However, the average size of these regions was about half as large as the one showr..

There are three regions of easterly shear to be considered in Fig. 13 and they have been numbered for reference. Climatology shows that October storms occurring in these regions in $55 \mathrm{y}$ zars number 14,2 and 1 , respectively. Temperature clifferences between surface and $500 \mathrm{mb}$, as accurately as they can be determined, are 33, 34 and $29 \frac{1}{2} \mathrm{C}$, respectively. Area 1 has a little stronger maximum shear than area 2, while area 3 has very weak shear. Area 3 can be eliminated and areas 1 and 2 are about even when strength of shear and lapse rate are weighted together. Climatology indicates that area 1 is more favorable for storm formation.

Fig. 14 shows four regions of easterly shear, all about the same size. July climatological statistics show that the number of storms which origina ied in these regions are $8,1,3$ and 0 , respectively. When the intertropical sea-level pressure-trough line is added (dashed-dotted line), it effectively eliminates region 3 from consideration, since storms do not come fro:n the region south of the trough. The climatological odds now favor region 1 very heavily.

Fig. 15 shows the simultaneous inception of two cyclonic disturbances. Both were bred in the large easterly shear region which has much stronger shear than does region 1 and is favored by climatology 44 to 13. Both regions have the same temperature difference from sea-level to $500 \mathrm{mb}$.

Three Pacific cases in different months of different years were analyzed and all of the disturbances were traced back to such regions of easterly shear. Observed winds were used in another Pacific case and again the result was the same. It appears to be necessary but not sufficient that easterly shear from $1000-500 \mathrm{mb}$ be 

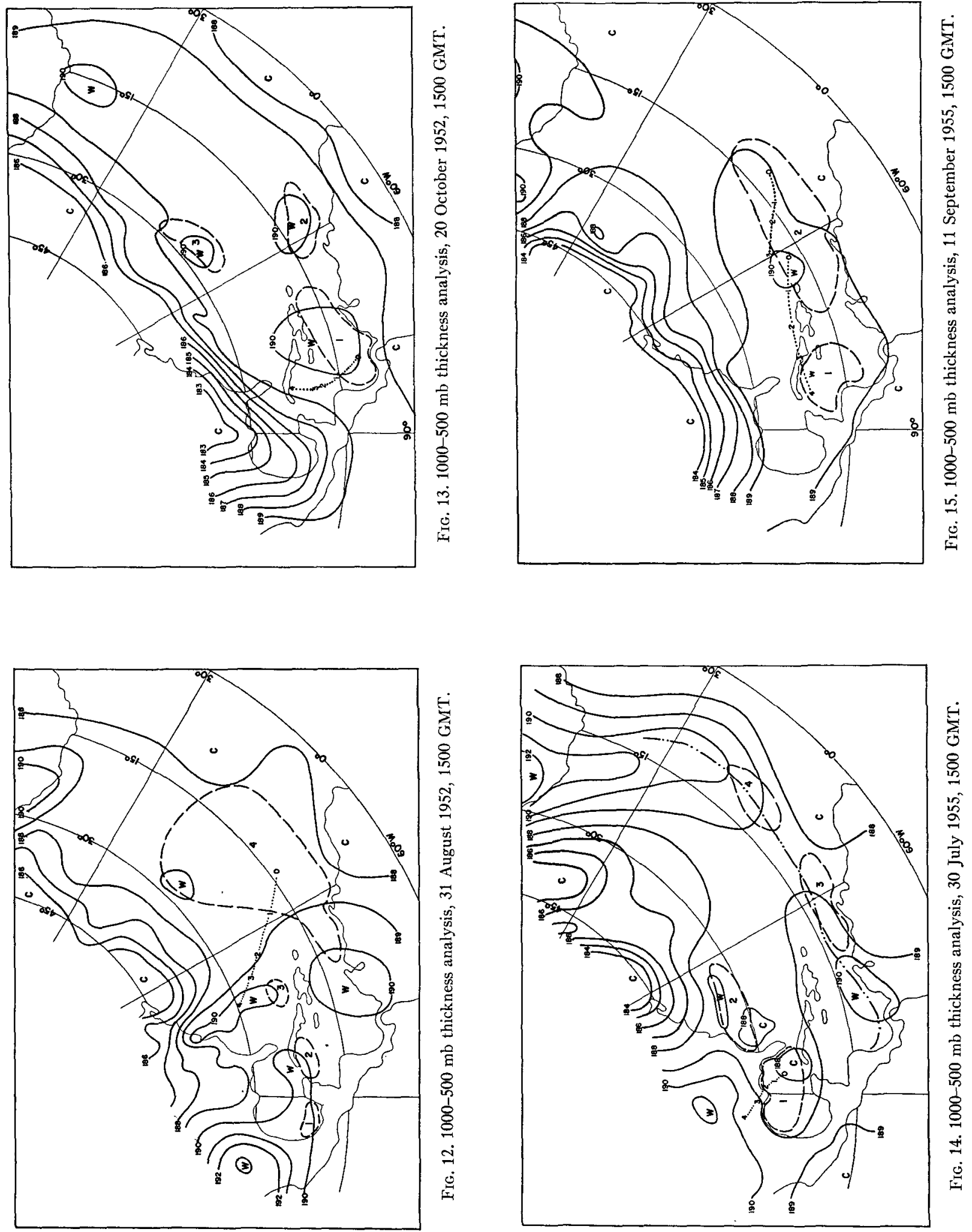


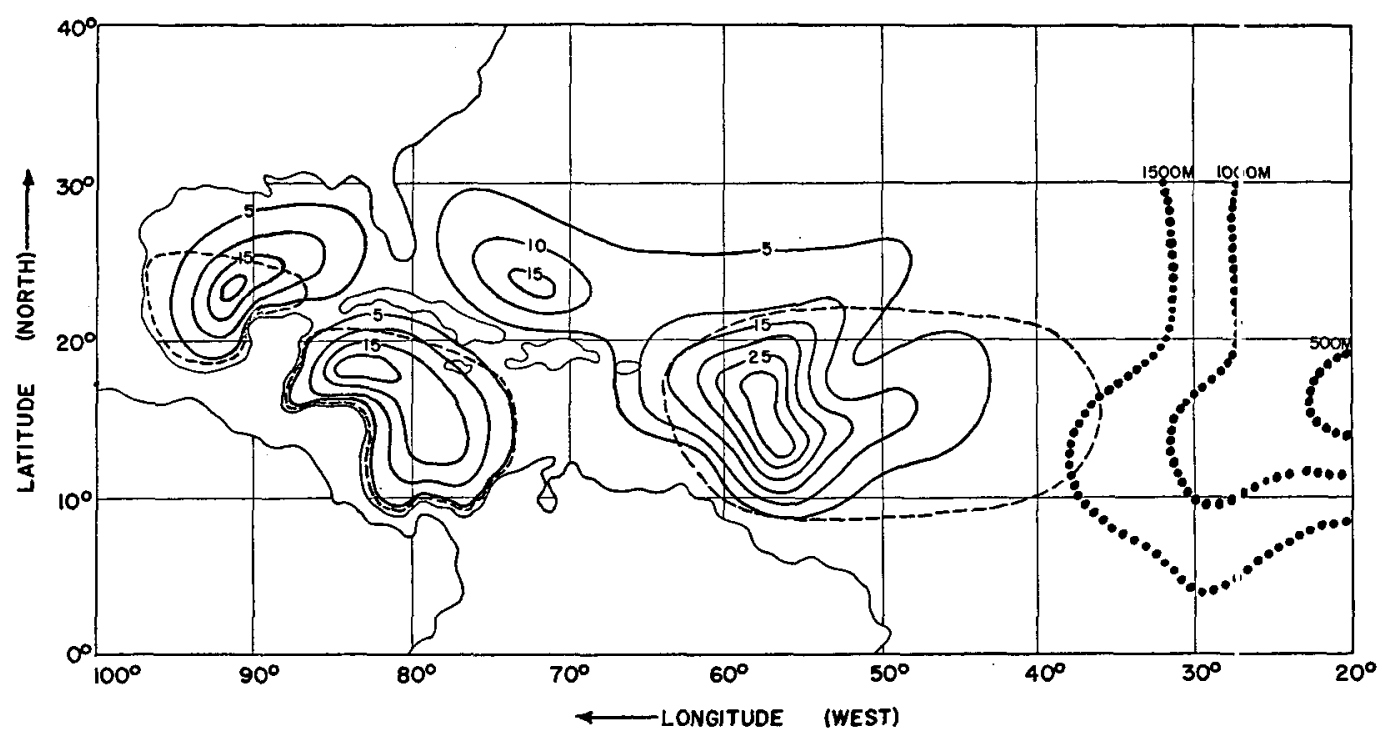

FIG. 16. Distribution of tropical cyclone formation during the years 1901 to 1955 .

present in areas of initial appearance of cyclonic disturbances.

A seasonal map, Fig. 16, was prepared from the tropical storm-track charts of Tannehill (1955) and includes all storms of hurricane and near hurricane intensity for the years 1901-1955 inclusive. The place of initial occurrence was taken as the beginning point on the track and totals for 5-deg lat-long squares were plotted and analyzed. The dotted lines enclose regions of net easterly wind shear from 3 to $10 \mathrm{~km}$. These regions were taken from a chart of the "pressure" thickness obtained from the U. S. Weather Bureau July normal 3- and 10-km maps (1944). Regions of easterly shear coincide quite well with the three main areas of maximum storm inception. The small maximum of storm occurrence north of western Hispaniola has no counterpart on the pressure-difference map. Since lateseason storms comprise this maximum, a good correlation with a July mean pressure-difference map should not be expected. The central Atlantic region of easterly shear (dashed line) extends eastward only as far as the 1500-m isopleth of trade-wind-inversion height. Storms occurring in the area of low mean-inversion height must originate when the inversion is absent or much displaced from the mean position. History shows that only 2 per cent of all storms develop in this region.

\section{Forecasting tropical disturbances}

The computation of the parameter which is related to the time of formation of waves (Fig. 2) is based on information gained from the dependent data of JulyAugust-September 1955. During that season the 500-mb ridge never moved south of $18 \mathrm{~N}$, the ridge height when averaged over $20 \mathrm{deg}$ of longitude, never exceeded 19,600 geopotential feet and the slope of the pressure (height) profile south of the ridge never exceeded $55 \mathrm{deg}$. (An ordinate value of 100 geopotential feet was made equivalent to an abscissa value of $5 \mathrm{deg}$ of latitude when the height profiles were plot:ed and the slopes obtained.) The parameter was computed in such a way that the three factors were added and weighted equally, and each factor was zero when the limiting condition was met. That is, if the ridge was at $18 \mathrm{~N}$, had a mean height of $19,600 \mathrm{ft}$ and a pressure-profile slope south of it equalling $55 \mathrm{deg}$, then the parameter and each of its component parts are zero. Very large values indicate high latitude, low height values in 'he ridge and weak pressure gradients to the south. All three factors were averaged over $20 \mathrm{deg}$ of longitude centered on the highpressure cell (or cells, if two were present).

The method was applied to independent data on a current basis at the National Hurricane Research Project Headquarters in West Palmı Beach, Fla., for a period of nearly one month in August and September

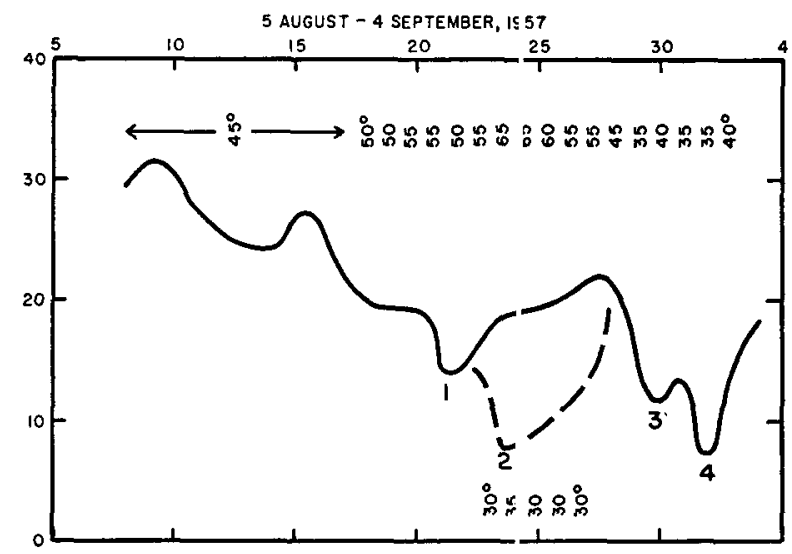

FIG. 17. Parameter related to time of tropical cyclone formation vs. time (1957). 
1957. The 500-mb ridge often tended to separate into two cells, or to change longitude when appearing as one cell. Longitudinal averaging for the timing parameter was shifted east or west with the high cell, and two curves were maintained when two distinct high cells existed. These curves are shown in Fig. 17, with the central longitude noted for each day on each curve. A forecast of a region of probable wave formation, as well as time, can be obtained from the curve and the surface and 500-mb maps. The initial trough should appear some distance WSW of the point of strongest low-level pressure gradient south of the high cell. The thickness analysis then shows if the wave will form in and/or move into a region favorable for development. Fig. 18 summarizes results of the test. Solid circles indicate places where waves were forecast, the forecast time being taken from the curves in Fig. 17. Solid lines indicate troughs which appeared in surface streamline analyses, and dashed lines connecting successive $24-\mathrm{hr}$ trough positions coincide with latitudes of largest streamline amplitude. A (V) on a trough line indicates that a closed vortex could be identified. Empty circles represent positions of observed waves at forecast times. The only wave which became an intense vortex was hurricane Carrie; it is shown by a series of crosses, and as a hurricane after the first advisory was issued. Each of the cases is discussed briefly below. The waves and the storm were extrapolated backwards from known positions to get the paths shown in Fig. 18.

At 0000 GMT 22 August, the curve in Fig. 17 dipped to a value of 14 and then began to rise. On the basis of the late August part of the 1955 curve (Fig. 2), a wave of fairly large amplitude was forecast. Later verification showed that the time and place of forecast formation were very close (compare solid and empty circles). The wave showed an identifiable vortex on 24 and 25 August but not after that, although the wave persisted.
Thickness analyses showed a net westerly shear (1000-500 mb) on 21 August where the wave formed, a very weak easterly shear at the wave position from the 22 nd to 25 th, and westerly again thereafter.

On 23 August a more easterly high cell appeared as the westerly one moved slowly northward. The curve based on the easterly cell dipped very low early on 24 August. The verification showed a wave forming almost exactly where it was forecast but about 12 hours earlier. This wave continued to move westward for a week or so but never developed an identifiable vortex. Thickness analyses showed weak easterly shear at the wave position from 23 to 26 August and westerly shear after the 26th.

A third wave was forecast late on 30 August when the parameter (now applied to the eastern high cell) dipped to a value of 12 . The wave, which appeared at the time and very near the place forecast, remained nearly stationary while its amplitude increased and then moved regularly westward. A vortex was indicated on 3 and 4 September, followed by a marked decrease in intensity. Thickness analyses initially showed a weak easterly shear, decreasing to about zero on the 31st and 1 st, becoming weak easterly again late on the 1st and westerly by the 4 th.

A pronounced drop in the parameter was noted on 1 September. A low value of 7 prompted the forecast of a vortex; and because the thickness pattern in the formation region was quite favorable for the first time during the test period, a potential hurricane was forecast on 1 September. On 6 September ship reports showed concrete evidence of hurricane-force winds, and hurricane Carrie persisted in the Atlantic for at least two weeks after that date. Backward extrapolation of the track of this vortex indicated that initial formation probably occurred very near to the place and time forecast.

All of the four cases noted above were forecast quite

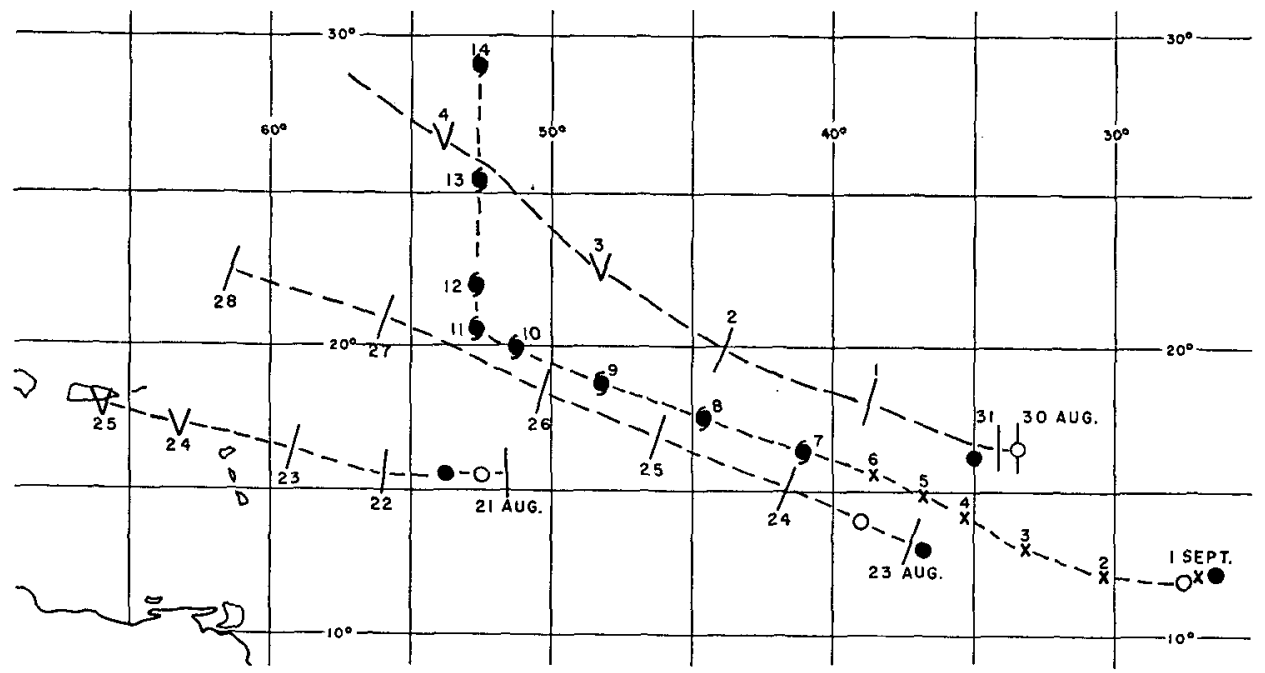

FIG. 18. Verification of forecasting method (1957). 
accurately (time and place of occurrence), certainly within the accuracy limits of the verification method. No cases of waves or vortices of low-level origin occurred which were not forecast during the test period. Changes in intensity also were accurately forecast.

\section{Concluding remarks}

Unstable waves tend to appear in the mid-Atlantic easterlies when the subtropical anticyclone is strong and south of its mean position. Changes in the intensity and position of the anticyclone are also closely related to changes in the westerly zonal index. Application of the vorticity equation to a normal wind map provides a possible physical explanation of the empirical findings.

Application of the Bjerknes-Holmboe theory to the easterlies indicated that low-level tropical waves and vortices require a net easterly vertical wind shear between 1000 and $500 \mathrm{mb}$ in order to develop into storms of hurricane intensity. In some 30 . cases, no exception to this rule was found.

A forecasting method was developed for predicting time and place of wave formation in the easterlies based on the position and strength of the subtropical high cell or cells. Intensification was forecast when a net easterly shear, $1000-500 \mathrm{mb}$, existed at wave position, rapid intensification if the shear was large. The dependent data of 1955 gave good results when the forecasting method was applied. Application to independent data resulted in quite accurate forecasting of the place and time of origin of four disturbances and of their future development. Waves or vortices which start at high levels and work downward or assist in development of very weak surface waves cannot be forecast by this method.
Finally, it would seem that the na:ure of and changes in the large scale circulation patterns of middle latitudes may have more influence than previously supposed on both the formation and development of tropical disturbances.

Acknowledgments. Thanks are cue to the U. S. Weather Bureau forecasting and research groups and the U. S. Navy forecasting group, all located in Miami, Fla., for assistance in the practical aspects of this work. Many persons in the Department of Meteorology at Florida State University assisted in some part of the work. The greatest individual contribution was by N. E. LaSeur, whose critical reading, of the manuscript and helpful suggestions during many discussions proved invaluable.

\section{REFERENCES}

Bjerknes, J., and J. Holmboe, 1944: On the theory of cyclones. J. Meteor., 1, 1-22.

Forsythe, G. E., 1945: A generalization of :he thermal wind equation to arbitrary horizontal flow. Bull. Amer. meter. Soc., 26, 371-375.

Godske, C. L., T. Bergeron, J. Bjerknes and R. C. Bundgaard, 1957: Dynamic meteorology and weat'ter forecasting. Boston, Amer. Meteor. Soc., 790 pp.

Namias, J., 1950: The index cycle and its role in the general circulation. J. Meteor., 7, 130-139.

Newton, C. W., 1959: Axial velocity streaks in the jet stream: ageostrophic "initial" oscillations. $J$. Meteor., 18, 638-645.

Riehl, H., T. C. Yeh and N. E. LaSeur, 1953: A study of variations of the general circulation. J. Meleor., 7, 181-194.

Tannehill, I. R., 1955: Hurricanes. Prin:eton, Princeton Univ. Press, $275 \mathrm{pp}$.

U. S. Weather Bureau, 1938: Allas of climatic charts of the oceans. Washington, D. C.

- 1944: Normal weather maps, Nortitern Hemisphere upper level. Washington, D. C.

$\longrightarrow, 1952,1953,1955:$ Synoptic weather maps. Washington, D. C. 